\title{
Hepatic Epiteloid Hemangioendothelioma, a Diagnosis to keep in mind when finding Incidentalomas
}

\section{Introduction}

Hepatic Epitheloid Hemangioendothelio$\mathrm{ma}(\mathrm{HEH})$ is considered a rare tumor with vascular origins that has an overexpression of vascular endothelial growth factor (VEGF) and its receptors VEGFR (Weiss SW, Enzinger FM. Cancer 1982 Sept; 50(5): 970-981). Until now, there has been no standardized treatment for this pathology, the only suitable treatment being surgery, including liver resection, liver transplantation, or considering recent studies, transcatheter arterial chemoembolization (Mehrabi A et al. Cancer 2006 Nov; 107: 2108-2121; Cardinal J et al. Arch Surg. 2009; 144: 1035-1039). The evolution of a HEH is unpredictable. Sometimes the tumor has a quiet and stable course but it can also evolve aggressively and become metastatic (Sangro B et al. Rare Tumors 2012 Apr; 4(2): e34).

The diagnosis of HEH is established first through imaging methods; it is shown as an hypoechoic tumor on ultrasonography (Lyburn ID et al. American Journal of Roentgenology 2003; 180: 1359-1364), with low density on CT, and on MRI usually exhibits low signal intensity on T1 weighted images and high signal intensity on T2 weighted images (Salech F et al. Ann Hepatol. 2011; 99-102, Ros LH et al. Canadian Association of Radiologists Journal. 1999; 387-389; Kehagias DT et al. Hepato-Gastroenterology. 2000; 1711-1713). The imaging findings of $\mathrm{HEH}$ have some typical features but have a size-dependent pattern with contrast enhancement, on both CT and MRI images (Lisha Z, et al. BMC Gastroenterol. 2015; DOI: doi: 10.1186/s12876-015-0299-x). HEH exhibits great heterogeneity regarding the imaging findings (Lyburn ID et al. American Journal of Roentgenology. 2003 May; vol.180: 1359-1364). Studies show that it can appear as a single or multiple avascular masses with calcification, and can involve the entire liver (den Bakker MA et al. Pathol Res Pract 1998; 194; Issue 3: 194-198; EH, Rha SE, Lee YJ et al.

\section{License terms}

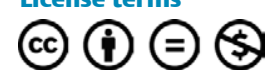

Abdom Imaging. 2015 Mar; 40(3): 500509). Some imaging suggestions have

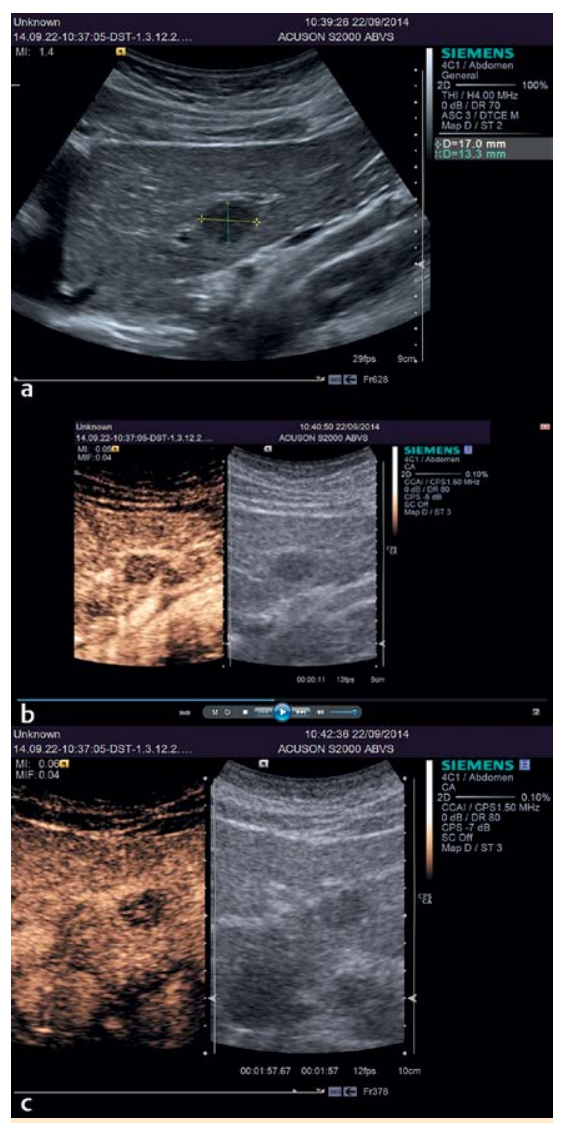

Fig. 1 CEUS enhancement pattern in the left hepatic lobe: a B mode, $\mathbf{b}$ Arterial phase, c Late phase.

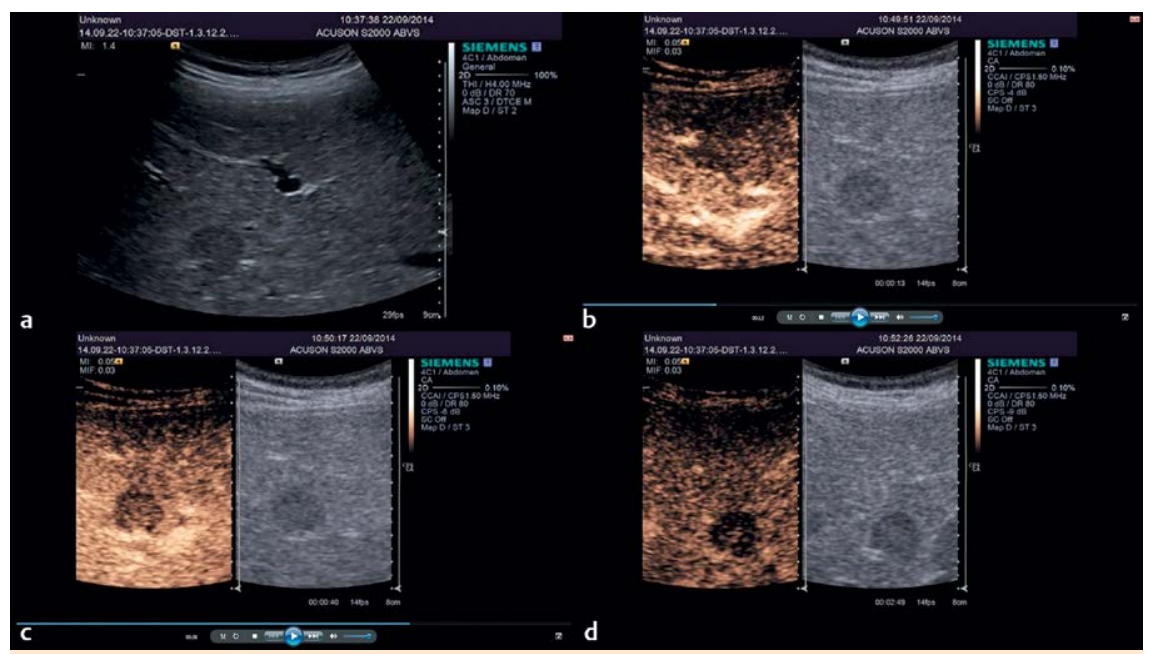

Fig. 2 CEUS enhancement pattern in the Right Hepatic Lobe: a B mode, b Arterial phase, c Portal phase, $\mathbf{d}$ Late phase. been proposed in order to improve diagnostic accuracy, such as the retraction sign (capsule retraction of the liver, near the lesion) (Miller WJ et al. American Journal of Roentgenology. 1992; 159: 53-57). Another is the halo sign, which is related to the i.v administration of contrast medium (a hyperintense layer between the hypointense center and periphery) ( Linand J, Ji Y. Hepatobiliary and Pancreatic Diseases International. 2010; 154-8), even though HEH is often misdiagnosed as being a metastatic tumor. In this report we shall present a case of a young woman diagnosed with multiple liver tumors that proved to be $\mathrm{HEH}$. We shall likewise discuss related imaging aspects.

\section{Case Presentation \\ $\nabla$}

A 27-year-old woman, asymptomatic, without other known pathology, was incidentally diagnosed with multiple liver masses after a routine abdominal ultrasonography. The clinical examination and biochemical tests did not reveal any abnormalities. Tumor markers including carcinogenic embryonic antigen (CEA), carbohydrate antigen 19-9 (CA19-9) and alpha-fetoprotein were also normal.

Baseline US showed multiple lesions, hypo-hyperechoic, ( $\bullet$ Fig. 1,2), with a maximum diameter of $20 \mathrm{~mm}$. CEUS examination revealed a "rim like" enhancement of the most representative lesion of the left hepatic lobe (LHL) ( $\bullet$ Fig. 1), with washout pattern in the portal and late phase. CEUS examination of the right hepatic lobe (RHL) lesion (the largest one) ( $\bullet$ Fig. 2). showed a slightly hyperenhanced pat- 


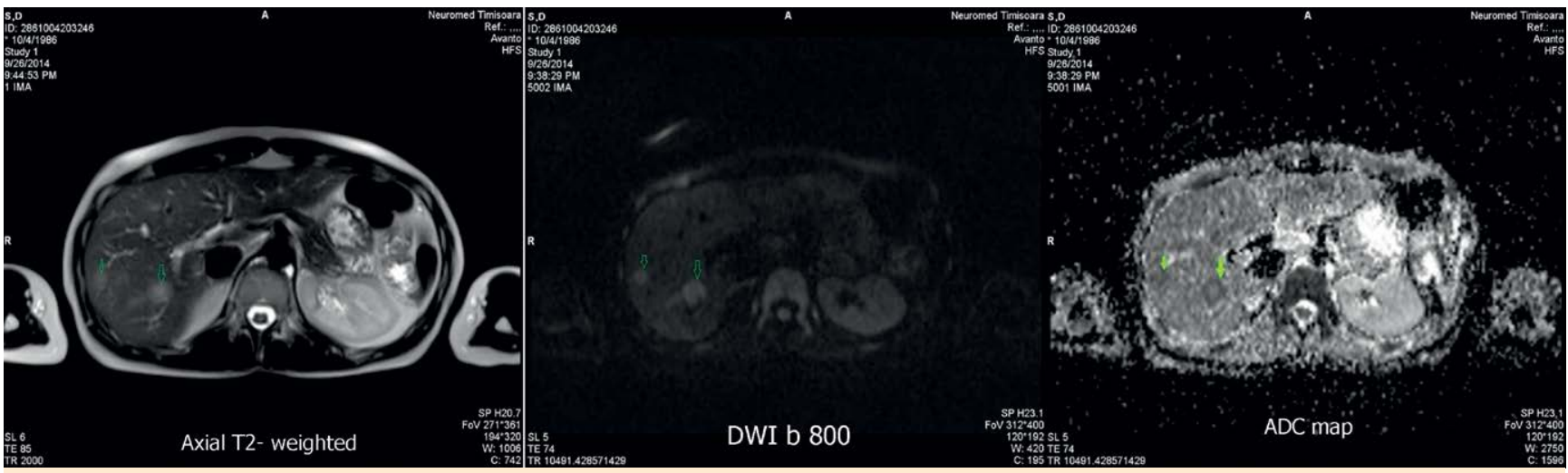

Fig. 3 The 2 nodular lesions (arrowheads), in axial T2 weighted image and ADC map, show target-like appearance with which the central region of the nodule showing higher intensity. On high b value DWI this lesions show restricted diffusion.

tern in the arterial phase, with evident washout in the portal and late phase, suggesting malignancy. Subsequently, an abdominal MRI confirmed multiple lesions (๑ Fig. 3), with discreet hyperintensity in $\mathrm{T} 2$, hypointensity in $\mathrm{T} 1$, and with restricted diffusion (diffusion coefficient of 0.96 - suggestive of malignancy) (॰ Fig. 3 ). Precontrast ( $\odot$ Fig. 4a) and ( $\bullet$ Fig. 4 b-d) dynamic postcontrast T1 weighted fatsaturated axial images at the same level showed slight peripheral ring-like enhancement in the arterial phase ( $\bullet$ Fig. 4b) and progressive enhancement during the portal venous phase ( $\bullet$ Fig. 4c) and delayed phase ( $\bullet$ Fig. 4d). In the delayed phase the lesions presented as a halo sign, thus confirming the literature (Paolantonio P, et al. J Magn Reson Imaging 2014; 40: 552-558). The same patient in a postcontrast image (axial portal venous and coronal delayed phase) at a superior level exhibited another subcapsular lesion producing retraction of the liver capsule ( $\odot$ Fig. 4 e-f).

The patient was also evaluated with transvaginal and thyroid ultrasound examinations which were unremarkable. A thorax CT was performed and demonstrated a small nodule $(12 \mathrm{~mm})$ in the right lung that could be characterized in the clinicalimaging context as metastatic.

Finally a core biopsy was performed that through immunohistochemistry staining disclosed the diagnosis of HEH. The biopsy indicated an infiltrating tumor resembling epitheloid cells that spread within sinusoid and small veins with marked atrophic hepatocyte. The cells had a pleomorphic aspect, some of them having intracytoplasmic vacuoles (containing red blood cells), mimicking the "signet ring", negative for AA-PAS coloration. Imunohistochemically the tumor cells stained positive for vascular markers CD31, CD34, and Factor VIII antigen, underlying the endothelial origin of the tumor. Demonstrating the histological and imunohistochemical features, the diagnosis of Hepatic Epitheloid Hemangioendothelioma was established ( $\bullet$ Fig. 5). With this diagnosis the patient was referred to the Oncology department.

\section{Discussion \\ $\nabla$}

Epitheloid hemangioendothelioma is an intermediate tumor between hemangioma and angiosarcoma indicative of recurrence and metastatic activity, and is the most aggressive member of hemangioendothelioma family (Weiss SW, Goldblum JR, Soft Tissue Tumors. $20085^{\text {th }}$ edition; 681-702). The diagnosis is based on histological and immunohistochemical findings. Histologically the cells have an epithelioid appearance, are mildly pleomorphic, and typically show intracytoplasmic vascular lumina which contain red blood cells. The intracytoplasmic lumina impart a "signet ring" appearance which sometimes may be mistaken for an adenocarcinoma. The presence of red blood cells differentiates them from signet ring cell adenocarcinoma which contains intracellular mucin. The tumor typically forms papillary tufts and glomeruloid structures within adjacent portal venules and sinusoidal spaces.

Regarding immunohistochemistry, the tumor is positive for Factor VIII, CD31, and CD34. Keratin may be also positive. The tumor is negative for mucin. Differential diagnosis can be made with: angiosarcoma, hemorrhagic hepatocellular carcinoma, diffuse metastatic disease in sinusoids and cholangiocarcinoma (Liang Cheng et al. Essentials of Anatomy c Pathology 2011, $3^{\text {rd }}$ edition: 45-17).
A review of literature from 1984 to 2005 with more than 400 cases demonstrates female predominance (3:2), and a median age of 42 years (Mehrabi A et al. Cancer. 2006: 107: 2108-2121). The most frequent symptom is upper quadrant pain $(49 \%)$, and in $81 \%$ of cases the tumor involves both liver lobes, lungs being the most frequent site of metastases (13\% of patients have metastases), although $63 \%$ of the patients were non-metastatic (Mehrabi A et al. Cancer. 2006; 107: 2108-2121). Keeping in mind the expression of vascular endothelial growth factor (VEGF) and its receptor (VEGFR) in $\mathrm{HEH}$ makes reasonable the use of antiangiogenic therapies (Salech F et al. Ann Hepatol 2011; 10: 99-102). More than 200 studies published about HEH in last years report results of antiangiogenic agents such as bevacizumab, lenalidomide, thalidomide and sorafenib with variable response rates (Bruno Sangro et al. Rare Tumors 2012 Apr 12; 4(2): e34). Some studies reveal the antiangiogenic effect of non-steroidal anti-inflammatory drugs that have been used alone or combined with other drugs in the treatment of $\mathrm{HEH}$ (Yousaf N. J Clin Oncol 2013; 31 suppl: abstr 10569). Therefore, antiangiogenic therapy might be a treatment option for HEH.

Hepatic epithelioid hemangioendothelioma $(\mathrm{HEH})$ is a rare tumor with variable malignant potential. The only curative treatment remains liver transplantation, extrahepatic manifestation not being a contraindication for surgery (Mehrabi A, et al. Cancer. 2006; 107: 2108-2121; Lerut JP et al. Ann Surg 2007; 246: 949957; Grotz TE, HPB (Oxford) 2010; 12: 546-553). According to (Komatsu $S$ et al. World J Gastroenterol 2014 July; 20 (26): 8729-8735) new therapy options can be taken into consideration, such as the two- 


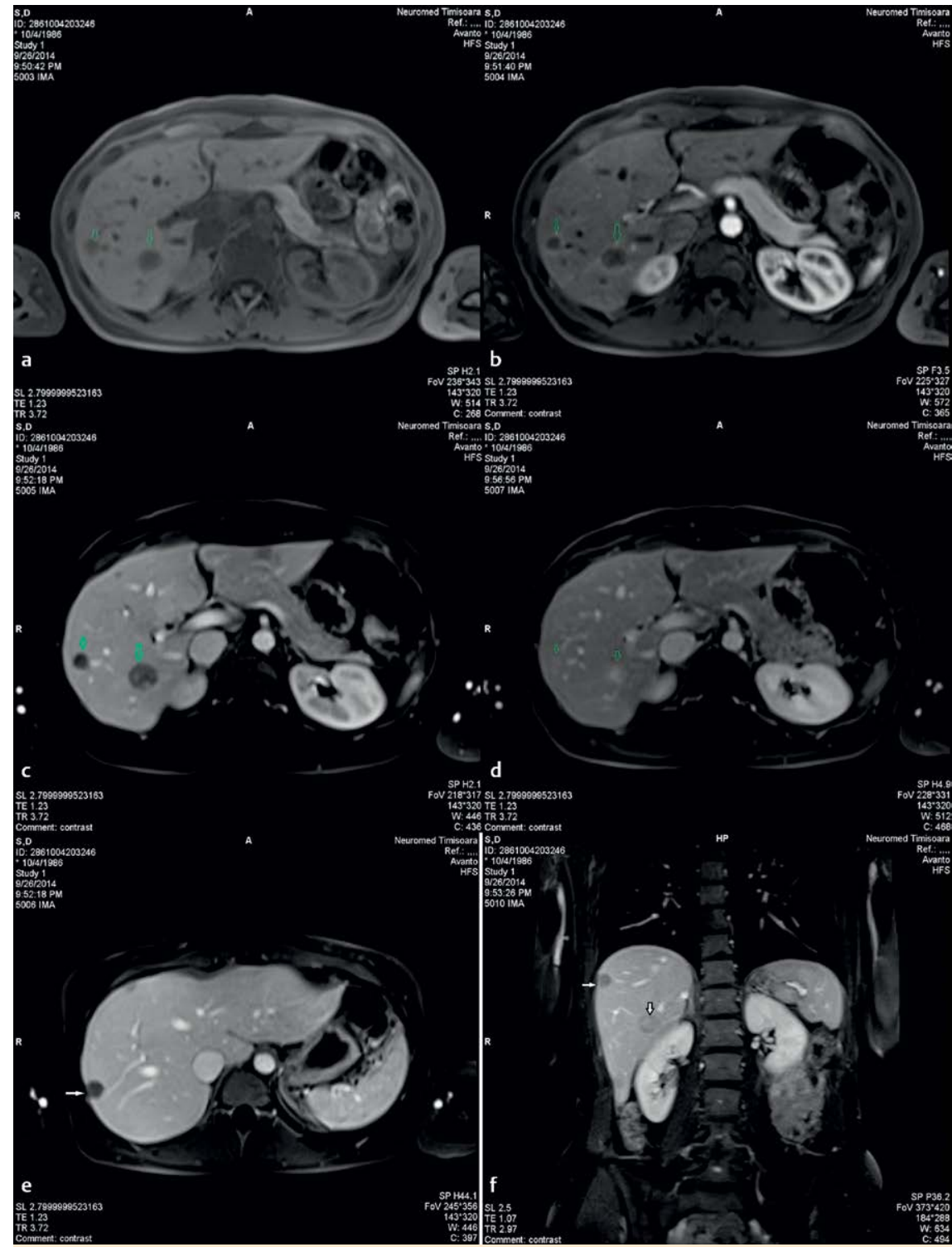

Fig. 4 Precontrast $\mathbf{a}$ and $\mathbf{b}$-d dynamic postcontrast $\mathrm{T} 1$ weighted fat-saturated axial images, show slight peripheral ring - like enhancement in arterial phase $\mathbf{b}$ and progressive enhancement during portal venous phase $\mathbf{c}$ and delayed phase $\mathbf{d}$, in delayed phase the lesions presenting as the halo-sign. Postcontrast image (axial portal venous and coronal delayed phase) a subcapsular lesion that produce retraction of the liver capsule (horizontal arrow in $\mathbf{e}$ and $\mathbf{f}$ ). The vertical arrow in $\mathbf{f}$ show the halo-sign (coronal delayed phase) in the one lesion situated at the same level with the lesions from previous images (a-d).

stage treatment, hepatectomy and carbon-ion therapy. This, along with the studies of Wang et al. (Wang LR, World J Surg 2012; 36: 2677-2683) and Cardinal et al. (Cardinal J et al. Arch Surg. 2009; 144: 1035-1039) underlining the effectiveness of TACE vs. hepatectomy, opens the possibility of expanding the curative treatment options for multiple bilobar hepatic tumors. Although we should not forget the natural course of the disease, some data from the literature (Makhlouf HR et al. Cancer 1999; 85: 562-582; Otrock ZK et al. Lancet Oncol 2006; 7: 439-441) showed a 5-year survival time of patients without any treatment, indi- cating the borderline malignant nature of the disease. In fact, our patient is currently under imaging and medical surveillance.

The particularity of this case is the clinical and biological silence of the disease. The initial imaging evaluation misleads the first diagnosis characterizing the lesions as being metastatic. Both imaging methods highlight the ring-like/rim-like enhancement and malignant pattern of the lesions, concluding the diagnostic algorithm with a core biopsy.

In conclusion we pinpoint the risk of misdiagnosing the pathology even in tertiary centers and the need for personalizing

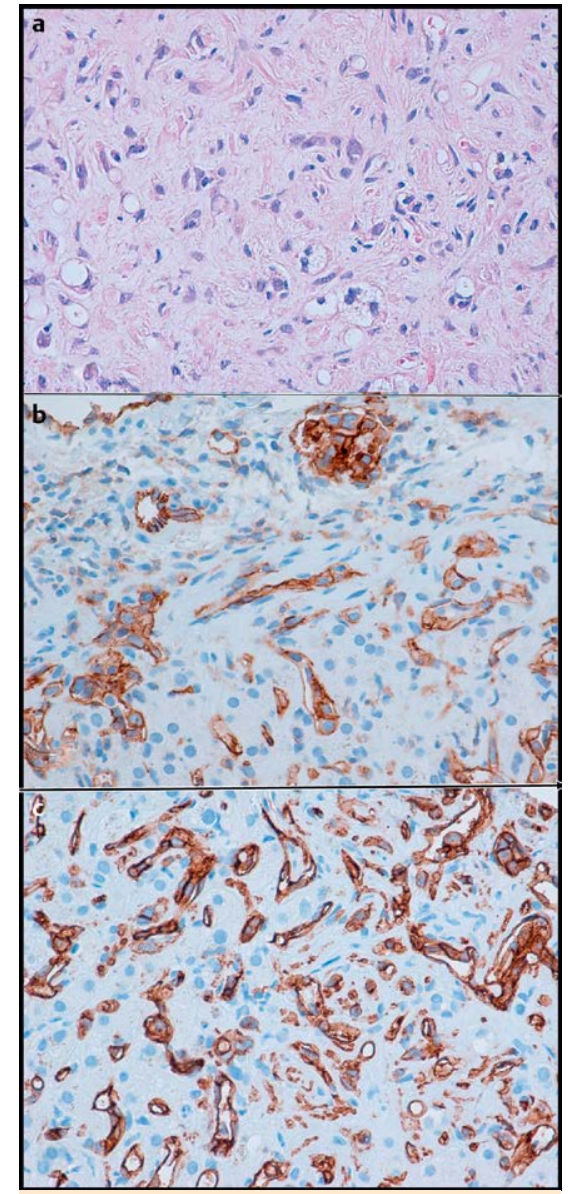

Fig. 5 Histological aspect: a Hematoxylin eosin staining, b CD31 staining, c CD34 staining.

treatment management according to each patient's characteristics and local medical experience.

\section{Acknowledgements}

$\nabla$

The research published in this paper was made with support from the grant INOVIAHEP awarded by the "Victor Babes" University of Medicine and Pharmacy Timisoara, in PROGRAMUL III - C2 - PCFI $-2015 / 2016$.

T. Moga, A. Popescu, S. C. Ivascu, M. Cornianu, I. Sporea 